\title{
A PROPOSITO Di EMANCIPARE: \\ «SE GLI ADULTI NON ASCOLTANO I BAMBINI \\ VANNO INCONTRO A GUAI GROSSI». \\ LA CITTÀ DEI BAMBINI: UNA NUOVA FILOSOFIA \\ DI GOVERNO DELLE CITTÀ
}

\author{
Francesco Tonucci \\ Istituto di Scienze e Tecnologie della Cognizione \\ del Consiglio Nazionale delle Ricerche (CNR) \\ responsabile del progetto internazionale \\ «La città delle bambine e dei bambini»
}

SOMMARIO: Emancipare significa rendere liberi, concedere autonomia. Nella città moderna, che si è sviluppata a misura dei cittadini adulti e delle loro automobili, i bambini hanno perso la possibilità di muoversi autonomamente e non vengono riconosciuti come cittadini, ma solo come futuri cittadini, bisognosi di cura e di protezione. Di fronte alle inadempienze degli adulti e ai «guai» che stanno producendo nel mondo contemporaneo, il progetto «La città delle bambine e dei bambini» propone una reale emancipazione infantile chiamando i bambini a partecipare al governo delle città e restituendo loro la possibilità di muoversi autonomamente negli spazi pubblici della città. Il progetto intende dare piena attuazione alla Convenzione dei diritti dell'Infanzia, approvata dalle Nazioni Unite nel 1989 e finora poco conosciuta e sostanzialmente inattuata. A conclusione del contributo si riferiscono alcuni esempi di come concrete proposte infantili possano modificare sostanzialmente la politica delle nostre città.

Parole Chiave: diritti dei bambini, partecipazione, autonomia di movimento, sicurezza urbana, spazio pubblico.

RESUMEN: Emancipar significa liberar, conceder autonomía. La ciudad moderna, se ha desarrollado a medida de los ciudadanos adultos y de sus automóviles, los niños han perdido la posibilidad de moverse de forma autónoma y 
no se les reconoce como ciudadanos, sino sólo como futuros ciudadanos, necesitados de cuidado y protección. Frente a los incumplimientos de los adultos y a los «líos» que están provocando en el mundo contemporáneo, el proyecto La Ciudad de las Niñas y los Niños propone una emancipación infantil real llamando a los niños a participar en el gobierno de las ciudades y devolviéndoles la posibilidad de moverse de manera autónoma en los espacios públicos de la ciudad. El proyecto pretende aplicar en su totalidad la Convención de los derechos de la Infancia, aprobada por las Naciones Unidas en 1989 y hasta el momento poco conocida y prácticamente sin aplicar. Al final del artículo se incluyen algunos ejemplos de cómo propuestas infantiles concretas podrían modificar sustancialmente la política de nuestras ciudades.

Palabras clave: derechos de los niños, participación, autonomía de movimiento, seguridad urbana, espacio público.

RESUM: Emancipar significa alliberar, concedir autonomia. La ciutat moderna s'ha desenvolupat a mida de la ciutadania adulta i dels automòbils, els xiquets i les xiquetes han perdut la possibilitat de moure's per la ciutat de manera autònoma i no se'ls reconeix com a ciutadans, sinó només com a futurs ciutadans que necessiten cura i protecció. Davant dels incompliments dels adults i els «embolics» que provoquen en el món contemporani, el projecte La Ciutat de les Xiquetes i dels Xiquets» proposa una emancipació infantil real i crida els xiquets a participar en el govern de les ciutats, per a retornar-los la possibilitat de moure's de manera autònoma als espais públics de la ciutat. El projecte pretén aplicar-hi totalment la Convenció dels Drets de la Infantesa, aprovada per les Nacions Unides, en 1989, i fins ara poc coneguda i pràcticament sense aplicar. Al final de l'article s'inclouen alguns exemples de com propostes infantils concretes podrien modificar substancialment la política urbana.

Paraules Clau: drets dels xiquets i xiquetes; participació; autonomia de moviment, seguretat urbana; espai públic.

ABSTRACT: Emancipating means freeing, granting autonomy. In the modern city, which has developed to accommodate adult citizens and their cars, children have lost the ability to move autonomously and they are not recognised 
as citizens, but rather only as future citizens, in need of care and protection. In light of adults' inadequacies and the 'mess' they are making in today's world, the City of Girls and Boys project calls for real emancipation for children, inviting them to participate in governing their cities and giving them back the ability to move autonomously in the city's public spaces. The aim of the project is to apply in full the Convention on the Rights of the Child approved by the United Nations in 1989 and to date, unfamiliar to most and yet to be implemented practically everywhere. The article concludes with some examples of how children's specific proposals can substantially alter the politics of our cities.

KEYWORDS: children's rights, participation, autonomy of movement, urban safety, public space.

Dici: è faticoso frequentare i bambini.

Hai ragione.

Aggiungi: perché bisogna mettersi al loro livello, abbassarsi, scendere, piegarsi, farsi piccoli.

Ti sbagli.

Non è questo l'aspetto più faticoso.

È piuttosto il fatto di essere costretti a elevarsi fino all'altezza dei loro sentimenti.

Di stiracchiarsi, allungarsi, sollevarsi sulle punte dei piedi.

Per non ferirli.

KORCZAK

E mancipare in italiano ha diversi significati: 1. Rendere liberi (una popolazione dal dominio straniero); 2. Liberare un figlio dalla patria potestà o uno schiavo facendolo libero (antica Roma); 3. Attribuire al minore di età, se ne esistono le condizioni, una limitata capacità di agire. ${ }^{1}$ Si utilizza questo verbo quindi per indicare la liberazione di un popolo dalla dominazione straniera o lo schiavo dal suo padrone rendendolo libero. Ma si utilizza anche nel caso dei bambini quando si concedono loro maggiori autonomie

1. Dal dizionario italiano Zingarelli. 
e libertà, essendo essi sottoposti al controllo e alla responsabilità dei loro genitori.

Da che mondo è mondo i bambini sono oggetto di protezione e cura da parte degli adulti che garantiscono così il loro diritto di vivere senza pericoli l'età più delicata e fragile della loro vita. L'età della preparazione, della crescita, dell'attesa. L'attesa di diventare adulti, assumere ruoli sociali riconosciuti. I bambini sono i futuri adulti, i futuri genitori, i futuri interpreti del mondo adulto, i futuri cittadini. Si preparano a diventare come i loro genitori, come i loro maestri, come i loro governanti.

In cambio i bambini, da che mondo è mondo, ricambiano gli adulti con l'obbedienza, l'ascolto, il rispetto.

Ma un bambino di 5 anni di una piccola città italiana, discutendo con la sua insegnante e con i suoi compagni della scuola dell'Infanzia sui diritti delle bambine e dei bambini, dice: «Se gli adulti non ascoltano i bambini vanno incontro a guai grossi»».

Severn Suzuki bambina canadese di 12 anni, nel vertice di Rio de Janeiro del 1992 davanti ai Capi di Governo di tutto il mondo disse: «I genitori dovrebbero poter confortare i loro figli dicendo loro: andrà bene, il mondo non finirà, noi stiamo facendo del nostro meglio. Ma io non credo che i nostri genitori possano più dirci queste cose. Ciò che voi fate mi fa piangere di notte».

Victoria, una bambina di 10 anni, del Consiglio dei bambini di Rosario in Argentina diceva: «La colpa di tutto è dei grandi. Bisogna mettere dei limiti ai grandi».

Questi tre bambini, in un modo particolarmente forte, violento, denunciano i loro genitori e gli adulti di non rispettare il loro ruolo, di non esercitare come sarebbe loro dovere la tutela che dovrebbero garantire ai loro figli e il piccolo di 5 anni suggerisce una soluzione: gli adulti dovrebbero ascoltare i bambini. Questo intervento vuole verificare la validità delle denunce di questi bambini e il valore delle loro proposte alla luce di una emancipazione dell'infanzia che significhi qualcosa di più che il riconoscerle maggiore libertà, anche alla luce della Convenzione dei diritti dell'Infanzia e dell'Adolescenza, che nel 2014 compie 25 anni. 


\section{Guai seri}

Il bambino di cinque anni probabilmente non sa molto dei guai che gli adulti stanno provocando ma, come scriveva nell'undicesimo secolo Mosè Maimonide: «Da quando non esistono più o non sono più riconosciuti i profeti e la profezia non si manifesta più in forma chiara, l'arte della profezia è affidata ai bambini e ai pazzi a cui bisogna prestare attenzione». E questi bambini che ho citato esercitano effettivamente una funzione profetica. Ed è assolutamente vero che gli adulti di tutto il mondo, e specialmente quelli del mondo più ricco e sviluppato, stanno provocando guai molto gravi, come probabilmente mai se ne erano creati. A livello ambientale stiamo lasciando per i nostri figli e nipoti un mondo peggiore di quello che abbiamo ricevuto dai nostri padri e nonni: stiamo compromettendo forse in maniera irreversibile l'ambiente, contaminando l'aria, deforestando aree sempre più vaste, provocando l'innalzamento del clima, distruggendo specie animali e vegetali, consumando in modo irreversibile energie non rinnovabili. Nonostante tutte le promesse e gli accordi internazionali le differenze fra il mondo della fame e quello della ricchezza stanno aumentando: mentre il mondo ricco diventa più ricco, nel mondo povero aumentano la fame e le malattie. Nonostante le drammatiche lezioni delle dittature e della guerra mondiale del diciannovesimo secolo i paesi democratici non rifiutano la guerra. I nostri governi nazionali portano avanti il degrado morale, politico e sociale. A livello economico siamo arrivati ad una crisi economica profonda e, se mi è consentito, ridicola. Ridicola perché è il frutto della scienza di tutti i più grandi economisti del mondo. Una crisi economica probabilmente ripetibile appena le condizioni lo permetteranno. I nostri adolescenti e i nostri giovani manifestano il loro disagio e la loro avversità al mondo che stiamo offrendo loro con azioni aggressive contro la città e contro gli altri come il vandalismo e il bullismo, o con azioni, ancora più gravi, contro se stessi, come l'abuso di alcol e droghe, fino al suicidio. È noto il fenomeno chiamato hikikomori in Giappone: si stima che più di un milione di giovani fra i 14 e i 30 anni, prevalentemente maschi, vivano chiusi nella loro stanza, alimentati dai loro genitori, senza studiare o lavorare davanti allo schermo del computer. Hanno scelto la realtà virtuale, rifiutando quella reale. 
Siamo arrivati, per la prima volta nella storia, a lasciare a chi verrà dopo di noi una speranza di vita inferiore alla nostra. Noi dai nostri nonni abbiamo avuto quasi 10 anni di vita di più; i nostri nipoti, secondo recenti ricerche, avranno una speranza di vita media inferiore alla nostra.

La città storica, rifiutando il modello medioevale del castello, poderoso, difeso, separato dal borgo dove vivono i contadini e gli artigiani, nasce simbolicamente attorno ad una piazza, dove si affacciano il palazzo di governo e la cattedrale e dove si svolge il mercato. La città come luogo di incontro e di scambio, come spazio condiviso. La città moderna, dopo l'ultima guerra mondiale, quando ha dovuto provvedere alla ricostruzione, ha preferito scegliere come cittadino di riferimento un maschio, adulto, lavoratore, adattando le sue nuove strutture e i suoi servizi al lavoro, principale occupazione di questo cittadino-parametro, e all'automobile, suo giocattolo preferito. Questa città finisce per dimenticare chi non è maschio, chi non è adulto e chi non è lavoratore e le donne, gli anziani e i bambini finiscono per scomparire dalle strade e dallo spazio pubblico della città.

\section{Quanto costa ai bambini questa città?}

Il cambiamento probabilmente più rilevante fra l'essere bambini qualche decina di anni fa e oggi è che oggi i bambini non possono più uscire di casa da soli e hanno perduto il tempo libero. Vengono sempre accompagnati e vigilati e tutto il loro tempo è organizzato fra la scuola al mattino, i compiti pomeridiani e corsi di lingue, sport o attività ricreative, ma sempre scuole, sempre dentro, sempre vigilati. Il tempo che rimane lo si passa davanti ad uno schermo.

Il questa condizione si crea un grande squilibrio fra un aumento improvviso e impressionante di autonomia dei bambini, fin dai primi anni, rispetto all'informazione e alla comunicazione (internet e il telefono cellulare), mentre scompare completamente l'autonomia di movimento. I bambini sanno tutto dei coccodrilli ma non sanno avvicinarsi ad una lucertola, possono parlare e vedersi attraverso skype con lo zio in Australia, ma non possono scendere in strada per giocare con un amico. 
Questa nuova condizione ha costi molto alti per le bambine e i bambini.

- Se non possono uscire di casa senza essere accompagnati i bambini non possono giocare e se non giocano non possono crescere. Non si può essere «accompagnati» a giocare, occorre «lasciare» $\mathrm{i}$ bambini giocare. Il gioco, che è sicuramente l'esperienza che incide di più sullo sviluppo dei primi anni di vita (i più importanti in assoluto) ha bisogno di sufficiente libertà e autonomia per poter essere correttamente vissuto.

- Se non possono uscire da soli, per giocare con i loro amici, non possono scaricare le energie fisiche così come il loro corpo richiede, con gravi conseguenze fisiche come il soprappeso e l'obesità infantili che rappresentano un grave allarme per la loro salute.

- Se non possono vivere le esperienze dell'avventura, della scoperta, dell'ostacolo, del piacere o della delusione, non riusciranno ad assimilare le regole e a costruirsi gli strumenti necessari per affrontare il mondo e diventare grandi e autonomi.

- Se non potranno sperimentare il rischio, man mano che ne hanno desiderio e opportunità, a due, quattro, otto, dieci anni, perché c'è sempre qualcuno che vigila e controlla, si accumulerà un desiderio e un bisogno sempre più grande di trasgressione che potrà essere soddisfatto solo quando raggiungeranno una sufficiente autonomia: quando avranno le chiavi di casa o una moto sotto il sedere. Tutto quindi viene rinviato all'adolescenza ma con un pericolo notevolmente maggiore. Le esperienze di bullismo, l'abuso di alcol e droghe, una sessualità precoce e non controllata, gli incidenti di moto e di auto (in Italia sono la prima causa di morte fino ai 26 anni), i suicidi giovanili, credo che più che essere fenomeni e drammi dell'adolescenza, siano coerenti conseguenze di errori educativi nel periodo infantile. Chi non ha potuto andare in bicicletta e sbucciarsi le ginocchia da bambino ha più probabilità di subire incidenti gravi in moto da adolescente, chi non ha potuto fumare una sigaretta di nascosto a 9-10 anni è più facile che senta l'attrazione di uno spinello a 14. 


\section{La convenzione dei diritti dell'infanzia}

Il 20 novembre del 1959 le Nazioni Unite approvarono la Dichiarazione dei diritti dei bambini. Il 20 novembre del 1989, sempre a New York, le Nazioni Unite approvarono la Convenzione dei diritti dell'Infanzia. Fra i due documenti esiste una differenza fondamentale e il secondo lo possiamo considerare una vera e propria dichiarazione di «emancipazione» dell'infanzia. Il primo documento, che nasce dopo il secondo conflitto mondiale, si preoccupa di difendere e tutelare l'infanzia, affermando i suoi diritti alla vita, alla salute, all'alimentazione, all'istruzione, i suoi diritti a non essere sfruttata, abusata, utilizzata per attività militari. È un forte monito al mondo adulto a non dimenticare i suoi doveri verso i suoi figli.

Nella Convenzione del 1989 ovviamente si confermano questi diritti e queste raccomandazioni, ma si aggiunge una nuova visione dell'infanzia: si riconoscono le bambine e i bambini, come cittadini. Non futuri adulti che vanno tutelati e preparati nella delicata fase preparatoria, non futuri cittadini, ma cittadini, titolari di diritti, fin dalla nascita. Questo avrebbe dovuto cambiare radicalmente il rapporto fra adulti e bambini, ma la Convenzione, dopo 25 anni dalla sua approvazione, e nonostante sia stata riconosciuta da quasi tutti i paesi del mondo, ${ }^{2}$ è ancora sostanzialmente sconosciuta e di conseguenza, ovviamente, inattuata. Qualcosa si è fatto nel campo della tutela, dell'intervento umanitario per diminuire la fame, la malattia e l'ignoranza, ma quasi nulla si è fatto per riconoscere la cittadinanza dei bambini.

Valga per tutti l'analisi di quanto prescrive l'articolo 12. Dice che i bambini hanno diritto ad esprimere la loro opinione ogni volta che si prendono decisioni che li riguardino, e che il parere dei bambini deve essere tenuto nel giusto conto. Questo significa che nell'89, nella autorevole sede delle Nazioni Unite, tutti gli adulti del mondo promisero a tutti i bambini del mondo che non avrebbero preso più nessuna decisione che li riguardasse senza consultarli. Una promessa impressionante, un impegno altissimo e di altissimo valore morale. Ma al contempo un'enorme bugia perché, per quel che mi risulta,

2. Non hanno adottato la Convenzione gli Stati Uniti d'America e la Somalia. Gli Stati Uniti non possono sottoscriverla finché in alcuni Stati resterà legale la pena di morte per minori di 18 anni. 
nelle famiglie, nelle scuole, negli ospedali, nelle città, gli adulti continuano a prendere decisioni che riguardano le bambine e i bambini senza chiedere il loro parere e, ovviamente, senza tenerne conto.

Di fronte alla facile obiezione, molto frequente, che però non ci sono solo i bambini e che bisogna tener conto anche dei diritti delle altre persone, che bisogna evitare che i bambini disturbino, ecc., l'articolo 3 chiarisce in modo definitivo che l'interesse del bambino va considerato prioritario. Questo significa che se entra in conflitto con diritti di altri ha la precedenza.

L'articolo 4 afferma che «Gli stati si impegnano ad adottare tutti i provvedimenti legislativi, amministrativi e altri, necessari per attuare i diritti riconosciuti dalla presente Convenzione» ma nulla è stato fatto in questo senso, almeno in Italia. Le leggi nazionali, i regolamenti comunali e condominiali continuano a proibire o limitare il gioco dei bambini, pur affermato chiaramente dall'articolo 31. I regolamenti scolastici non prevedono strumenti di consultazione degli alunni, e così via.

Infine l'articolo 42 afferma «Gli Stati parti si impegnano a far largamente conoscere i principi e le disposizioni della presente Convenzione, con mezzi attivi e adeguati sia agli adulti che ai bambini». E anche in questo caso non è stato fatto praticamente nulla. La Convenzione è sconosciuta in quasi tutti gli ambienti sociali, sia in quelli amministrativi che in quelli politici ed educativi. È quindi difficile sperare che possa essere attuata e rispettata.

\section{I bambini possono aiutarci: il progetto «La città delle bambine e dei bambini»»}

Abbiamo ottenuto questi risultati, abbiamo provocato questi «guai seri» razionalmente, utilizzando tutte le conoscenze scientifiche disponibili, tutte le indicazioni della ricerca scientifica e tutti i poteri dei Paesi politicamente ed economicamente più forti. Consapevolmente, razionalmente, scientificamente stiamo rovinando il mondo. È difficile che, da soli e razionalmente, possiamo e sappiamo uscirne. Dobbiamo farci aiutare e dobbiamo inventare qualcosa di nuovo, affidarci alla fantasia e alla creatività.

Se siamo convinti che un cambiamento è necessario e urgente i bambini possono aiutarci. Ma dobbiamo essere disposti ad ascoltarli e capaci di capirli. 
Per questo sono necessarie alcune condizioni: essere convinti che abbiano cose importanti da dirci, saper capire quel che ci chiedono anche al di là di quel che ci dicono e avere il coraggio di tener conto di quello che chiedono, costi quel che costi .

Il progetto «La città delle bambine e dei bambini» propone ai sindaci, ai politici, agli amministratori, ma anche agli educatori (genitori e insegnanti) di chiedere aiuto e consiglio ai bambini. Di considerare i bambini come parametro di valutazione e di cambiamento per le città, pensando che una città adatta ai bambini sia una città migliore per tutti ${ }^{3}$. I bambini quindi non vengono chiamati come una delle tante categorie sociali o generazionali, ma come capaci di rappresentare l'»Altro», il diverso, il lontano dal potere, dalla competenza, dalla opinione comune, omogenea e conformista dell'adulto. Il bambino, quindi, come paradigma della diversità: il sindaco che impara ad ascoltare i bambini diventa realmente il sindaco di tutti.

Questa è la vera e definitiva emancipazione dei bambini: il riconoscerli cittadini capaci di partecipare e di contribuire al governo della loro città.

Un aspetto particolarmente emozionante è notare come le proposte di cambiamento urbano dei bambini coincidano sostanzialmente con quelle degli esperti e degli scienziati e in particolare degli psicologi, degli ambientalisti, dei sociologi, degli urbanisti, dei pediatri e anche dei giuristi e come invece si allontanino dalle scelte dei politici e degli amministratori delle città.

Il progetto si articola su due assi principali:

a. La partecipazione dei bambini al governo delle città attraverso i Consigli dei bambini, considerati come organi consultivi dei sindaci e delle Amministrazioni locali, come applicazione corretta dell'art. 12 della Convenzione del 1989, e la progettazione di spazi e arredi urbani partecipata dai bambini.

b. La restituzione ai bambini del diritto di muoversi liberamente nella propria città, rivedendo le politiche della mobilità, per permettere ai bambini le attività indispensabili del gioco, dell'esplorazione, dell'av-

3. Per una migliore conoscenza delle motivazioni, delle proposte e delle esperienze del progetto si possono consultare i volumi Tonucci (1996), e Tonucci (2002), e il sito web www.lacittadeibambini.org. 
ventura. La presenza dei bambini negli spazi urbani restituirà sicurezza alle città. Con questo obiettivo si promuove l'esperienza «A scuola ci andiamo da soli, con gli amici» a partire dai sei anni.

Il progetto ha promosso l'adesione in varie città e oggi ha una rete di più di 200 città in Italia, Spagna, Argentina, Messico, Colombia, Uruguay, Cile e recentemente in Libano.

\section{Una politica diversa}

Nei quasi venti anni di esperienza abbiamo raccolto le proposte dei bambini di vari paesi e queste concordano in alcune richieste chiave, che descrivono un'altra politica. I bambini chiedono ai loro governanti una politica diversa, più vicina ai bisogni e ai desideri di tutti i cittadini, più sensibile alle esigenze di sostenibilità ambientale e più economica. Una politica diversa da quella dei politici, che miri non al consenso, ma alla felicità.

\section{a. Una diversa politica della sicurezza}

La sicurezza è oggi considerata un tema centrale nel dibattito politico. Di fronte ad un paventato grave pericolo incombente la politica tranquillizza gli elettori dicendo: non vi preoccupate, ci pensiamo noi. E l'unico modo in cui si sta intervenendo è aumentando la difesa: polizia, telecamere, porte blindate, strumenti elettronici a lettura satellitare, telefoni cellulari. Qui si aprono varie contraddizioni. La più importante è il paradosso creato dalla diminuzione dei reati, come documenta il Ministero degli interni e come confermano gli amministratori delle grandi città italiane da un lato, e l'aumento della paura dall'altro. Il paradosso che ne deriva è che, come hanno sempre spiegato $i$ sociologi e gli psicologi sociali, se aumenta la difesa aumenterà di conseguenza la paura o la sensazione di pericolo. Il problema reale è probabilmente legato al fatto che oggi la paura è considerata una risorsa, sia dalla politica che la usa per aumentare il consenso elettorale, sia della comunicazione mediatica che dedica ai fatti più efferati di cronaca nera un'attenzione morbosa, che aumenta l'audience e, di conseguenza, gli introiti pubblicitari.

Si crea così un circolo vizioso che crea una sensazione di insicurezza che pagano principalmente le categorie più deboli, e per primi le bambine e $\mathrm{i}$ bambini. 
Qual è la proposta alternativa che avanzano i bambini, qual è la loro idea di sicurezza? Possiamo considerarla rappresentata dalla proposta di un bambino del Consiglio dei bambini di Rosario in Argentina: «Gli adulti ci devono aiutare, ma da lontano». Una proposta che meriterebbe una riflessione approfondita da parte di noi adulti. Aiutare da lontano certamente significa non accompagnare per mano, non controllare direttamente e personalmente il proprio figlio. Probabilmente significa impegnarsi per creare condizioni sociali (non personali) di accoglienza e di attenzione. Probabilmente può essere rappresentata dall'eloquente proposta di Herman, sempre di Rosario: «È facile (essere sicuri): basta che ci siano due adulti, che prendono un caffè, in ogni isolato». Non quindi in strada per vigilare i propri figli, ma per prendere un caffè. L'importante è esserci, perché la presenza rende sicura la strada.

A questa diversa idea di sicurezza se ne aggiunge un'altra ancor più innovativa e sconcertante: i bambini per strada rendono sicura la strada. Di nuovo un paradosso: non lasciamo uscire di casa i nostri figli perché riteniamo che la strada sia pericolosa e invece la strada è pericolosa perché non ci sono più $i$ bambini. Se ci sono i bambini noi siamo migliori. I bambini sanno risvegliare nei vicini del quartiere atteggiamenti di solidarietà e di attenzione, sanno costruire un nuovo vicinato.

Valga come documento a riprova di questa affermazione l'esperienza di Buenos Aires. Nel 2001, dopo l'ennesimo atto di violenza contro i bambini che andavano a scuola, in uno dei quartieri periferici della città la gente si ribella e si riunisce per decidere cosa fare. Rifiuta la richiesta di maggiore presenza di polizia (si possono verificare sparatorie con esiti imprevedibili) e decidono di far andare i bambini a scuola da soli, facendo riferimento al nostro progetto «La città dei bambini». Per farlo coinvolgono, come previsto, commercianti e anziani, sensibilizzano le scuole e i quartieri e chiamano questa esperienza «Percorsi sicuri verso la scuola». Nel 2005, quando l'esperienza si era ormai diffusa in molti quartieri della Gran Buenos Aires ed era entrata anche i alcuni quartieri della Capital Federal, il responsabile della sicurezza della città, in un convegno pubblico, dichiarò che nei distretti che promuovevano questa esperienza gli episodi di criminalità urbana erano diminuiti del 50\%! 


\section{b. Una diversa politica della mobilità e della salute}

Abbiamo già descritto le gravi conseguenze della mancanza di autonomia e di movimento autonomo delle bambine e dei bambini nella loro città. Se i bambini non possono sfogare liberamente le loro energie per un tempo adeguato ogni giorno corrono molti rischi per la loro salute e per il loro sviluppo. Non sono sufficienti le attività sportive a cui le famiglie li iscrivono nel pomeriggio, anche con gravi oneri economici. Queste attività non sono giochi ma scuole. Il movimento è controllato da un istruttore e finalizzato alla formazione di un futuro «campione» e quindi mai spontaneo e libero, ma controllato e diretto. Molte delle patologie infantili hanno la loro radice e causa nella prolungata inattività davanti ad uno schermo. In queste ore i bambini mangiano e bevono prodotti inadatti alla loro salute. Da tutte le ricerche finora condotte sembra che l'unica proposta vincente contro la televisione o i videogiochi, sia la possibilità di uscire di casa per andare a giocare liberamente con gli amici. L'autonomia di movimento è la vera grande prevenzione. Recenti ricerche dimostrano che anche sui disturbi dell'attenzione, che stanno provocando un preoccupante e ingiustificato bombardamento farmacologico sui nostri bambini, l'attività fisica e la mobilità autonoma hanno effetti significativi (Vinther D. 2012). Una città democratica e preoccupata della salute dei suoi cittadini, a cominciare dai più piccoli, dovrebbe garantire a tutti di potersi muovere liberamente nella propria città.

\section{c. Una diversa politica dello spazio pubblico e del gioco}

I bambini non vogliono spazi per bambini, luoghi separati e dedicati a loro dove passare del tempo sotto una continua sorveglianza di adulti. I giardinetti per bambini, con gli scivoli, le altalene o altri giochi sono un'invenzione moderna che non tiene conto dei desideri e delle necessità dei bambini. Per loro lo spazio giusto per giocare è lo spazio pubblico, a partire dalle scale e dal cortile di casa fino ai marciapiedi, alle piazze e ai giardini della città. Un città che voglia rispettare le esigenze dei bambini dovrebbe evitare di spendere soldi in questi spazi separati e stereotipati e favorire la presenza delle bambine e dei bambini negli spazi pubblici. 
Anche su questo i bambini hanno idee chiare e, dal lavoro da loro svolto in Consigli dei bambini italiani, spagnoli e argentini, emergono queste proposte che potrebbero diventare un programma di progettazione degli spazi urbani.

«Ci sono troppi parcheggi e i bambini non hanno spazio per giocare. Proponiamo di fare a metà: metà per le macchine e metà per i bambini»;

«Non spazi dedicati e specializzati, ma condivisi, e sicuri perché occupati»;

«Non serve la polizia»;

«Non ci dovrebbero essere i genitori»;

«Gli spazi di gioco sono tutti orizzontali e non ci si può nascondere»;

«I grandi mettono sempre gli stessi giochi nei giardinetti e non c'è gusto perché

è come vedere sempre lo stesso film; non c'è sorpresa»;

«I grandi mettono le aiuole nei giardini per non far giocare i bambini»;

«Dovrebbero metterci i cespugli così possiamo baciarci di nascosto»;

«Un posto, per essere adeguato ai bambini non dovrebbe essere troppo sicuro».

Interessante quest'ultima affermazione: il bambino non dice che non deve essere sicuro ma non «troppo» sicuro, come se l'eccessiva sicurezza togliesse la possibilità di gioco e di divertimento. ${ }^{4}$

Diritto al gioco. In questa città, nella quale i bambini possono utilizzare gli spazi pubblici come tutti gli altri cittadini si deve riconoscere il diritto al gioco. Questo significa innanzitutto che il gioco non può e non deve essere proibito. Essendo riconosciuto dall'articolo 31 della Convenzione dei diritti delle bambine e dei bambini, non può essere proibito né nelle case, né nelle città. Non si può proibire ai bambini di giocare sulle scale, negli androni o nei cortili dei condomini; non si può proibire di giocare sui marciapiedi o nelle piazze delle città. Dovrebbero scomparire le proibizioni e i cartelli di divieto. Sarebbe auspicabile che comparissero cartelli che dicano: «I bambini sono invitati a giocare»; «Il Comune rispetta il diritto al gioco»; «Gli adulti non devono disturbare i bambini che giocano».

L'altra condizione è che i bambini abbiano tempo per giocare. Questo significa principalmente meno compiti e meno attività pomeridiane. I bambini passano a scuola un numero di ore simile o superiore a quello che i loro

4. Alla domanda «Che cosa è il gioco per un bambino?» la neuropsichiatria infantile Françoise Dolto risponde: «Direi che è godere della realizzazione di un desiderio attraverso dei rischi». 
genitori passano al lavoro. È assurdo che la scuola senta il bisogno di impegnare anche le ore pomeridiane, quelle del fine settimana e delle vacanze. D'altra parte anche le famiglie dovrebbero fare un passo indietro, non occupare tutto il tempo rimanente con corsi e scuole pomeridiane di lingua, sport o attività espressive e lasciare ogni giorno ai propri figli un sufficiente tempo libero che possano amministrare autonomamente, insieme ai loro amici, in spazi da loro scelti. Naturalmente, come sempre è stato, questa autonomia dovrà essere vissuta all'interno di una cornice di regole di tempo e di spazio che le famiglie stesse indicheranno.

\section{d. Una diversa politica economica}

Kofi Annan, il Segretario Generale delle Nazioni Unite, l'8 maggio 2002 a New York, aprendo la Sessione Speciale dell'onu per l'Infanzia, chiudeva il suo intervento dicendo: «Come potremo fallire, soprattutto ora che sappiamo che ogni dollaro investito nel migliorare le condizioni dell'infanzia ha un ritorno per tutta la società di ben 7 dollari?» L'affermazione era sconcertante e veniva presentata di fronte a tutti i capi di stato e di governo del mondo. In tempi in cui la rendita dei capitali non raggiungeva mai il $10 \%$ si proponeva un tipo di investimento virtuoso che poteva rendere il $700 \%$ !

Qualche anno dopo ho scoperto da dove aveva tratto quell'informazione Kofi Annan, quando ho scoperto le ricerche di James Heckman, premio Nobel per l'economia nel 2000. Heckman aveva effettuato una ricerca in cui aveva selezionato un gruppo di bambini provenienti da quartieri popolari con alto tasso di criminalità e con un quoziente di intelligenza inferiore alla media $\mathrm{e}$ aveva offerto loro di frequentare per tre anni (dai 3 ai 6) una scuola infantile di alta qualità (negli Stati Uniti molto cara e, quindi, riservata solo alle classi sociali culturalmente ed economicamente più alte). Quando il campione sperimentale ha compiuto vent'anni lo studioso ha valutato i risultati e ha riscontrato che quei giovani avevano avuto una carriera scolastica e professionale molto più alta dei loro compagni di condizione sociale che non avevano partecipato all'esperimento e avevano avuto rispetto a loro una attività criminale del $70 \%$ più bassa. Non considerando il valore morale e sociale del risultato l'economista poté dimostrare che, valutando i costi e i benefici, la più alta produttività e il più basso costo sociale (polizia, servizi sociali, ospedali, car- 
ceri), si poteva dimostrare che ogni dollaro investito aveva reso più di 7 dollari e che, in una proiezione sull'intera vita dei soggetti, si poteva calcolare che la rendita potesse superare di molto gli 8 dollari.

Con queste basi scientifiche mi sento autorizzato a segnalare tre settori nei quali tutte le risorse investite potranno produrre forti rendimenti:

a. Allattamento naturale: garantire a tutte le madri di poter godere per almeno un anno dei loro figli e ad ogni bambino di poter godere di sua madre e, soprattutto, dell'allattamento. Per questo vale la pena modificare le garanzie pubbliche e le regole lavorative.

b. Gioco libero per bambine e bambini: creare le condizioni urbanistiche e un'organizzazione sociale affinché tutti i bambini possano godere di un tempo libero quotidiano durante il quale giocare con gli amici nei luoghi pubblici della città senza essere accompagnati da adulti.

c. Una scuola infantile di alto livello per tutte le bambine e i bambini.

Il Paese che avesse il coraggio di intervenire con queste misure uscirebbe dalla crisi economica in pochi anni e preparerebbe per i suoi cittadini un futuro pieno di speranza.

\section{Note bibliografiche}

Ader, J. e Jouve, H. (1991): «Jeu et contexte urbain». Architecture \& Comportement, 7, 115-119.

Aavv(2014):, Participación infantil y construcción de la ciudadanía, Graó, Barcelona.

Bateson, G. (1956): The Message "This Is Play», Josiah Macy Jr. Foundation, New York.

Bauman, Z. (2001): The Individualized Society, Polity Press, Cambridge.

BLAKely, K. S. (1994): ««Parents» Conceptions of Social Dangers to Children in the Urban Environment», Children's Environments, 11, 16-25.

Bozzo, L. (1995): «Il gioco e la città», Paesaggio urbano, 2, 30-33.

CaIllois, R. (1967): Les jeux et les hommes, Gallimard, Paris.

Chawla, L. (2001): Growing up in an Urbanizing World, Unesco Publishing/ Earthscan, Paris/London. 
De Saint Exupéry, A. (1943): Le Petit Prince, Gallimard, Paris.

Dolto, F. (2000): Il bambino e la città, Mondadori, Milano.

HART, R. (1997): Children's Participation: The Theory and Practice of Involving Young Citizens in Community Development and Environmental Care, Earthscan Publication Limited, London.

Prezza M.; F. R. Alparone, D.; Renzi e A. Pietrobono (2010): «Social Participation and Independent Mobility in Children: The Effects of two Implementations of «We Go to School Alone»», Journal of Prevention \& Intervention in the Community, 38 (1), 8-25.

Prezza, M.; S. Pilloni; C. Morabito; C. Sersante e F. R. Alparone (2000): «La mobilità autonoma dei bambini nel contesto urbano e il cortile, il parco e la strada privata come spazi di gioco», Psicologia della salute, 3, 81-97.

Rissotto, A. e F. Tonucci (2002): «Freedom of movement and environmental knowledge in elementary school children», Journal of Environmental Psychology, 22, 65-77.

Tonucci, F. (1996): La città dei bambini, Laterza, Bari. [(1997): La ciudad de los niños, Fundación Germán Sánchez Ruipérez, Madrid.]

Tonucci, F. (2002): Se i bambini dicono: adesso basta!, Laterza, Bari. [(2003): Cuando los niños dicen ;Basta!, Fundación Germán Sánchez Ruipérez, Madrid.]

TonuccI, F. (2005): «Citizen Child: Play as Welfare Parameter for Urban Life», Topoi, 24: 183-195.

Tonucci, F. e P. Natalini (2006): A scuola ci andiamo da soli, Gangemi Editore, Roma.

Tonucci, F.; D. Renzi e A. Prisco (2011): Il Consiglio dei bambini, Booklab, Roma.

Vinther, D. (2012): «Children who Walk to School Concentrate Better», ScienceNordic.com 
